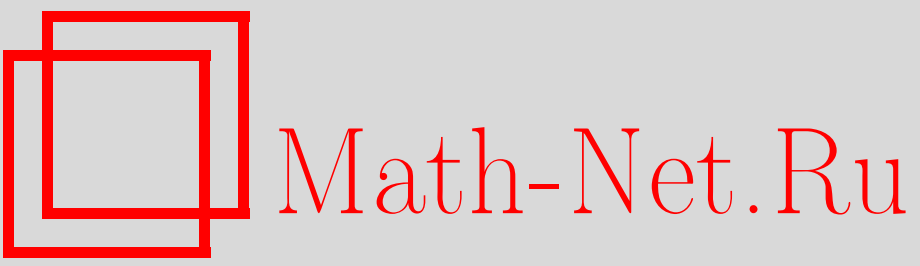

Э. А. Надарая, П. Бабилуа, Г. А. Сохадзе, Об интегральной квадратической мере уклонения одной непараметрической оценки бернуллиевской регрессии, Теория вероятн. и ее примен., 2012, том 57, выпуск 2, 322-336

DOI: https://doi.org/10.4213/tvp4449

Использование Общероссийского математического портала Math-Net.Ru подразумевает, что вы прочитали и согласны с пользовательским соглашением

http://www . mathnet.ru/rus/agreement

Параметры загрузки:

IP: 54.210 .77 .194

26 апреля 2023 г., 16:03:40 


\title{
ОБ ИНТЕГРАЛЬНОЙ КВАДРАТИЧЕСКОЙ МЕРЕ УКЛОНЕНИЯ ОДНОЙ НЕПАРАМЕТРИЧЕСКОЙ ОЦЕНКИ БЕРНУЛЛИЕВСКОЙ РЕГРЕССИИ
}

\begin{abstract}
В работе устанавливается предельное распределение интегрального квадратического уклонения непараметрической оценки типа ядра бернуллиевской функций регрессии. Построен критерий проверки гипотезы о бернуллиевской функции регрессии. Изучен вопрос состоятельности, и для некоторых сближающихся альтернатив исследована асимптотика поведения мощности.
\end{abstract}

Ключевые слова и фразы: бернуллиевская функция регрессии, мощность критерия, состоятельность, предельное распределение, плотность распределения.

Введение. Пусть случайная величина $Y$ принимает два значения 1 и 0 с вероятностями («успеха») $p$ и («неуспеха») $1-p$. Предположим, что вероятность «успеха» $p$ является функцией от независимой переменной $x \in[0,1]$, т.е. $p=p(x)=\mathbf{P}\{Y=1 \mid x\}$ (см. [1]-[3]). Пусть $x_{i}, i=$ $1, \ldots, n,-$ точки деления интервала $[0,1]$ :

$$
x_{i}=\frac{2 i-1}{2 n}, \quad i=1, \ldots, n .
$$

Пусть, далее, $Y_{i}, i=1, \ldots, n,-$ независимые бернуллиевские случайные величины с $\mathbf{P}\left\{Y_{i}=1 \mid x_{i}\right\}=p\left(x_{i}\right), \mathbf{P}\left\{Y_{i}=0 \mid x_{i}\right\}=1-p\left(x_{i}\right)$. Задача заключается в оценивании функции $p(x), x \in[0,1]$, по выборке $Y_{1}, Y_{2}, \ldots, Y_{n}$. Такая задача возникает, например, в биологии [1], [3], в области коррозионных исследований [4] и т.д.

В качестве оценки для $p(x)$ рассмотрим следующую статистику (см. [5], [6]):

$$
\begin{aligned}
\widehat{p}_{n}(x) & =p_{1 n}(x) p_{2 n}^{-1}(x) \\
p_{\nu n}(x) & =\frac{1}{n b_{n}} \sum_{i=1}^{n} K\left(\frac{x-x_{i}}{b_{n}}\right) Y_{i}^{2-\nu}, \quad \nu=1,2,
\end{aligned}
$$

*Тбилисский государственный университет им. Ив. Джавахишвили, факультет точных и естественных наук, ул. Университетская, 3, 0143 Тбилиси, Грузия; e-mail: elizbar.nadaraya@tsu.ge; petre.babilua@tsu.ge; grigol.sokhadze@tsu.ge 
где $K(x)$ - некоторая плотность распределения, удовлетворяющая сформулированным ниже требованиям, и $b_{n} \rightarrow 0-$ последовательность положительных чисел.

1. Предположения и обозначения. Предположим, что ядро $K(x) \geqslant 0$ выбрано так, чтобы оно было функцией с ограниченным изменением и удовлетворяло условиям: $K(x)=K(-x), K(x)=0$ при $|x| \geqslant \tau>0, \int K(x) d x=1$. Класс таких функций обозначим через $H(\tau)$.

Пусть $C^{(i)}$ обозначает множество функций $p(x), 0 \leqslant p(x) \leqslant 1, x \in$ $[0,1]$, имеющих ограниченные производные до $i$-го порядка, $i=1,2$.

Введем также следующие обозначения:

$$
\begin{gathered}
U_{n}=n b_{n} \int_{\Omega_{n}(\tau)}\left[p_{1 n}(x)-\mathbf{E} p_{1 n}(x)\right]^{2} d x, \quad \Omega_{n}(\tau)=\left[\tau b_{n}, 1-\tau b_{n}\right], \\
T_{n}=n b_{n} \int_{\Omega_{n}(\tau)}\left[\widehat{p}_{n}(x)-p(x)\right]^{2} p_{2 n}^{2}(x) d x, \\
Q_{i j}=\psi_{n}\left(x_{i}, x_{j}\right), \quad \psi_{n}(u, v)=\int_{\Omega_{n}(\tau)} K\left(\frac{x-u}{b_{n}}\right) K\left(\frac{x-v}{b_{n}}\right) d x, \quad p_{i}=p\left(x_{i}\right), \quad i=1, \ldots, n, \\
\sigma_{n}^{2}=4\left(n b_{n}\right)^{-2} \sum_{k=2}^{n} p_{k}\left(1-p_{k}\right) \sum_{i=1}^{k-1} p_{i}\left(1-p_{i}\right) Q_{i k}^{2}, \quad \varepsilon_{i}=Y_{i}-p\left(x_{i}\right), \\
\eta_{i j}^{(n)}=\frac{2 \varepsilon_{i} \varepsilon_{j} Q_{i j}}{n b_{n} \sigma_{n}}, \quad \varepsilon_{k}, \quad \xi_{1}^{(n)}=0, \quad k>n, \\
\xi_{k}^{(n)}=\sum_{i=1}^{k-1} \eta_{i k}^{(n)}, \quad k=2, \ldots, n, \quad \mathscr{F}_{k}^{(n)}=\sigma\left(\omega: \varepsilon_{1}, \ldots, \varepsilon_{k}\right),
\end{gathered}
$$

т.е. $\mathscr{F}_{k}^{(n)}$ есть $\sigma$-алгебра, порождённая случайными величинами $\varepsilon_{1}, \ldots, \varepsilon_{k}$, и $\mathscr{F}_{0}^{(n)}=(\varnothing, \Omega)$ (в дальнейшем для простоты записи вместо $\xi_{k}^{(n)}$ и $\eta_{i j}^{(n)}$ используем обозначения $\xi_{k}$ и $\left.\eta_{i j}\right)$.

\section{2. Вспомогательные утверждения.}

Лемма 1. Стохастическая последовательность $\left(\xi_{k}, \mathscr{F}_{k}\right)_{k \geqslant 1}$ является мартингал-разностью.

Лемма 2. Пусть $K(x) \in H(\tau)$ и $p(x), 0 \leqslant x \leqslant 1$, является также функиией е ограниченным изменением. Если $n b_{n} \rightarrow \infty$, то

$$
\begin{aligned}
& \frac{1}{n b_{n}} \sum_{i=1}^{n} K^{\nu_{1}}\left(\frac{x-x_{i}}{b_{n}}\right) K^{\nu_{2}}\left(\frac{y-x_{i}}{b_{n}}\right) p^{\nu_{3}}\left(x_{i}\right) \\
& \quad=\frac{1}{b_{n}} \int_{0}^{1} K^{\nu_{1}}\left(\frac{x-u}{b_{n}}\right) K^{\nu_{2}}\left(\frac{y-u}{b_{n}}\right) p^{\nu_{3}}(u) d u+O\left(\frac{1}{n b_{n}}\right)
\end{aligned}
$$

равномерно по $x, y \in[0,1]$, где $\nu_{i} \in \mathbf{N} \cup\{0\}, i=1,2,3$. 
Д о к а з а т е л ь с т в о соотношения (1) аналогично доказательству утверждения леммы 1 из [7, п. 1, лемма 1 , формула (2), с. 1643].

Лемма 3. Пусть $K(x) \in H(\tau)$ u $p(x) \in C^{(1)}$. Eсли $n b_{n}^{2} \rightarrow \infty$, mo

$$
b_{n}^{-1} \sigma_{n}^{2} \longrightarrow \sigma^{2}(p)=2 \int_{0}^{1} p^{2}(x)(1-p(x))^{2} d x \int_{|x| \leqslant 2 \tau} K_{0}^{2}(x) d x
$$

$u$

$$
\Delta_{n}=\mathbf{E} U_{n}=\Delta(p)+O\left(b_{n}\right)+O\left(\frac{1}{n b_{n}}\right)
$$

гдe

$$
\Delta(p)=\int_{0}^{1} p(x)(1-p(x)) d x \int_{|x| \leqslant \tau} K^{2}(x) d x, \quad K_{0}=K * K .
$$

Д о к а з а т ел ь с т в о. Имеем

$$
\begin{aligned}
\sigma_{n}^{2} & =2\left(n b_{n}\right)^{-2}\left\{\sum_{k, i=1}^{n} p_{k}\left(1-p_{k}\right) p_{i}\left(1-p_{i}\right) Q_{i k}^{2}-\sum_{i=1}^{n} p_{i}^{2}\left(1-p_{i}\right)^{2} Q_{i i}^{2}\right\} \\
& =d_{1}(n)+d_{2}(n), \quad p_{i}=p\left(x_{i}\right), \quad i=1, \ldots, n .
\end{aligned}
$$

Легко видеть, что

$$
b_{n}^{-1}\left|d_{2}(n)\right|=2 n^{-2} b_{n}^{-3} \sum_{i=1}^{n} p_{i}^{2}\left(1-p_{i}\right)^{2}\left(\int_{\Omega_{n}(\tau)} K^{2}\left(\frac{x-x_{i}}{b_{n}}\right) d x\right)^{2} \leqslant c_{1} \frac{1}{n b_{n}}
$$

Далее, пользуясь определением $Q_{k i}$, получаем

$$
\begin{gathered}
d_{1}(n)=\frac{2}{\left(n b_{n}\right)^{2}} \int_{\Omega_{n}(\tau)} \int_{\Omega_{n}(\tau)}\left(\sum_{i=1}^{n} p\left(x_{i}\right)\left(1-p\left(x_{i}\right)\right) K\left(\frac{x-x_{i}}{b_{n}}\right)\right. \\
\left.\times K\left(\frac{y-x_{i}}{b_{n}}\right)\right)^{2} d x d y .
\end{gathered}
$$

Согласно лемме 2, отсюда имеем

$$
\begin{aligned}
d_{1}(n)=2 \int_{\Omega_{n}(\tau)} \int_{\Omega_{n}(\tau)}\{ & \frac{1}{b_{n}} \int_{0}^{1} p(u)(1-p(u)) \\
& \left.\times K\left(\frac{x-u}{b_{n}}\right) K\left(\frac{y-u}{b_{n}}\right) d u\right\}^{2} d x d y+O\left(\frac{1}{n b_{n}}\right) \\
=2 \int_{\Omega_{n}(\tau)} \int_{\Omega_{n}(\tau)}\{ & \int_{(x-1) / b_{n}}^{x / b_{n}} p\left(x-u b_{n}\right)\left(1-p\left(x-u b_{n}\right)\right) \\
\times & \left.K(u) K\left(\frac{y-x}{b_{n}}-u\right) d u\right\}^{2} d x d y+O\left(\frac{1}{n b_{n}}\right) .
\end{aligned}
$$


Учитывая, что $p(x) \in C^{(1)}$ и $\left[(x-1) / b_{n}, x / b_{n}\right] \supset[-\tau, \tau]$ для всех $x \in \Omega_{n}(\tau)$, из (6) находим, что

$d_{1}(n)=2 \int_{\Omega_{n}(\tau)} \int_{\Omega_{n}(\tau)} p^{2}(x)(1-p(x))^{2} K_{0}^{2}\left(\frac{x-y}{b_{n}}\right) d x d y+O\left(b_{n}^{2}\right)+O\left(\frac{1}{n b_{n}}\right)$.

Без особого труда можно установить, что

$$
\begin{aligned}
b_{n}^{-1} d_{1}(n)= & 2 \int_{0}^{1} p^{2}(x)(1-p(x))^{2}\left(\int_{(x-1) / b_{n}+\tau}^{x / b_{n}-\tau} K_{0}^{2}(u) d u\right) d x \\
& +O\left(b_{n}\right)+O\left(\frac{1}{n b_{n}^{2}}\right) .
\end{aligned}
$$

Стало быть,

$$
b_{n}^{-1} d_{1}(n) \longrightarrow 2 \int_{0}^{1} p^{2}(x)(1-p(x))^{2} d x \int_{|x| \leqslant 2 \tau} K_{0}^{2}(x) d x .
$$

Из (5) и (7) следует утверждение (2).

Теперь докажем (3). Имеем

$$
\mathbf{D} p_{1 n}(x)=\frac{1}{\left(n b_{n}\right)^{2}} \sum_{i=1}^{n} K^{2}\left(\frac{x-x_{i}}{b_{n}}\right) p\left(x_{i}\right)\left(1-p\left(x_{i}\right)\right) .
$$

В силу леммы 2 , отсюда находим

$$
\mathbf{D} p_{1 n}(x)=\frac{1}{n b_{n}^{2}} \int_{0}^{1} K^{2}\left(\frac{x-u}{n}\right) p(u)(1-p(u)) d u+\frac{1}{\left(n b_{n}\right)^{2}} .
$$

Далее, учитывая, что $\left[(x-1) / b_{n}, x / b_{n}\right] \supset[-\tau, \tau]$ для всех $x \in \Omega_{n}(\tau)$, из (8) получаем

$$
\mathbf{D} p_{1 n}(x)=\frac{1}{n b_{n}} p(x)(1-p(x)) \int_{|u| \leqslant \tau} K^{2}(u) d u+O\left(\frac{1}{n}\right)+O\left(\frac{1}{\left(n b_{n}\right)^{2}}\right) .
$$

Следовательно,

$$
\mathbf{E} U_{n}=\int_{0}^{1} p(x)(1-p(x)) d x \int_{|u| \leqslant \tau} K^{2}(u) d u+O\left(b_{n}\right)+O\left(\frac{1}{n b_{n}}\right) .
$$

Лемма 3 доказана.

3. Асимптотическая нормальность статистик $U_{n}$ и $T_{n}$. Имеет место следующее утверждение.

Теорема 1. Пусть $K(x) \in H(\tau)$ u $p(x) \in C^{(1)}$. Если $n b_{n}^{2} \rightarrow \infty$, mo

$$
\frac{b_{n}^{-1 / 2}\left(U_{n}-\Delta(p)\right)}{\sigma(p)} \stackrel{d}{\longrightarrow} N(0,1)
$$

где $\Delta(p)$ и $\sigma(p)$ определены в лемме $3 u \stackrel{d}{\longrightarrow}$ обозначает сходимость по распределению, а $N(0,1)$ - случайную величину, имеющую стандартное нормальное распределение $\Phi(x)$. 
Д о к а з а т ел ь с т в о. Имеем

$$
\frac{U_{n}-\Delta_{n}}{\sigma_{n}}=H_{n}^{(1)}+H_{n}^{(2)},
$$

где

$$
H_{n}^{(1)}=\sum_{k=1}^{n} \xi_{k}, \quad H_{n}^{(2)}=\frac{\sum_{i=1}^{n}\left(\varepsilon_{i}^{2}-\mathbf{E} \varepsilon_{i}^{2}\right) Q_{i i}}{n b_{n} \sigma_{n}} .
$$

Покажем, что $H_{n}^{(2)}$ сходится к нулю по вероятности. В самом деле,

$$
\mathbf{D} H_{n}^{(2)} \leqslant \frac{1}{\left(n b_{n} \sigma_{n}\right)^{2}}\left[3 \sum_{i=1}^{n}\left(p_{i}^{2}\left(1-p_{i}\right)^{2}+p_{i}\left(1-p_{i}\right)\left(1-6 p_{i}\left(1-p_{i}\right)\right)\right)\right] Q_{i i}^{2},
$$

где $p_{i}=p\left(x_{i}\right)$.

Так как $Q_{i j} \leqslant c_{2} b_{n}$ и $b_{n}^{-1} \sigma_{n}^{2} \rightarrow \sigma^{2}(p)>0$ при $n \rightarrow \infty$, то отсюда следует, что

$$
\mathbf{D} H_{n}^{(2)} \leqslant c_{3} \frac{1}{n b_{n}} \longrightarrow 0
$$

Стало быть, $H_{n}^{(2)} \stackrel{\mathbf{P}}{\longrightarrow} 0$ (здесь и ниже $\stackrel{\mathbf{P}}{\longrightarrow}$ обозначает сходимость по вероятности).

Теперь покажем, что $H_{n}^{(1)} \stackrel{d}{\longrightarrow} N(0,1)$. С этой целью проверим, что применимы следствия 2 и 6 теоремы 2 из работы [8]. Нужно доказать выполнение имеющихся в этих утверждениях условий, гарантирующих асимптотическую нормальность квадратично интегрируемой мартингал-разности, чем, согласно лемме 1 , является наша последовательность $\left(\xi_{k}, \mathscr{F}_{k}\right)_{k \geqslant 1}$.

Непосредственный подсчет показывает, что $\sum_{k=1}^{n} \mathbf{E} \xi_{k}^{2}=1$. Асимптотическая нормальность будет иметь место, если при $n \rightarrow \infty$

$$
\sum_{k=1}^{n} \mathbf{E}\left[\xi_{k}^{2} I\left(\left|\xi_{k}\right| \geqslant \varepsilon\right) \mid \mathscr{F}_{k-1}\right] \stackrel{\mathbf{P}}{\longrightarrow} 0
$$

и

$$
\sum_{k=1}^{n} \xi_{k}^{2} \stackrel{\mathbf{P}}{\longrightarrow} 1
$$

В [8] доказано, что при выполнении (10) и условия $\sup _{1 \leqslant k \leqslant n}\left|\xi_{k}\right| \stackrel{\mathbf{P}}{\longrightarrow} 0$ имеет место и условие $(9)$.

Поскольку при $\varepsilon>0$

$$
\mathbf{P}\left\{\sup _{1 \leqslant k \leqslant n}\left|\xi_{k}\right| \geqslant \varepsilon\right\} \leqslant \varepsilon^{-4} \sum_{k=1}^{n} \mathbf{E} \xi_{k}^{4},
$$

то, согласно приводимому ниже соотношению (11), для доказательства

$$
H_{n}^{(1)} \stackrel{d}{\longrightarrow} N(0,1)
$$


нам остается проверить лишь (10). Для этого достаточно убедиться в том, что

$$
\mathbf{E}\left(\sum_{k=1}^{n} \xi_{k}^{2}-1\right)^{2} \longrightarrow 0 \quad \text { при } n \rightarrow \infty,
$$

т.е., поскольку $\sum_{k=1}^{n} \mathbf{E} \xi_{k}^{2}=1$, что

$$
\mathbf{E}\left(\sum_{k=1}^{n} \xi_{k}^{2}\right)^{2}=\sum_{k=1}^{n} \mathbf{E} \xi_{k}^{4}+2 \sum_{1 \leqslant k_{1}<k_{2} \leqslant n} \mathbf{E} \xi_{k_{1}}^{2} \xi_{k_{2}}^{2} \longrightarrow 1 .
$$

В первую очередь установим, что

$$
\sum_{k=1}^{n} \mathbf{E} \xi_{k}^{4} \longrightarrow 0 \quad \text { при } n \rightarrow \infty \text {. }
$$

Принимая во внимание определения $\xi_{k}$ и $\eta_{i j}$, напишем

$$
\sum_{k=1}^{n} \mathbf{E} \xi_{k}^{4}=I_{n}^{(1)}+I_{n}^{(2)}
$$

где

$$
\begin{aligned}
I_{n}^{(1)} & =\frac{16}{\left(n b_{n}\right)^{4} \sigma_{n}^{4}} \sum_{k=2}^{n} \mathbf{E} \epsilon_{k}^{4} \sum_{j=1}^{k-1} \mathbf{E} \epsilon_{j}^{4} Q_{j k}^{4}, \\
I_{n}^{(2)} & =\frac{48}{\left(n b_{n}\right)^{4} \sigma_{n}^{4}} \sum_{k=2}^{n} \sum_{i \neq j} \mathbf{E} \epsilon_{j}^{2} \mathbf{E} \epsilon_{i}^{2} Q_{j k}^{2} Q_{i k}^{2} .
\end{aligned}
$$

Поскольку

$$
\begin{gathered}
Q_{i j} \leqslant c_{3} b_{n}, \quad \mathbf{E} \epsilon_{j}^{4}=\left(1-p_{i}\right)^{4} p_{i}+p_{i}^{4}\left(1-p_{i}\right) \leqslant c_{4}, \\
\mathbf{E} \epsilon_{j}^{2} \leqslant \frac{1}{4} \quad \text { и } \quad b_{n}^{-1} \sigma_{n}^{2} \rightarrow \sigma^{2}(p),
\end{gathered}
$$

то $I_{n}^{(1)}=O\left(1 /\left(n b_{n}\right)^{2}\right), I_{n}^{(2)}=O\left(1 /\left(n b_{n}^{2}\right)\right)$. Стало быть,

$$
\sum_{k=1}^{n} \mathbf{E} \xi_{k}^{4} \longrightarrow 0 \quad \text { при } n \rightarrow \infty \text {. }
$$

Покажем теперь, что

$$
2 \sum_{1 \leqslant k_{1}<k_{2} \leqslant n} \mathbf{E} \xi_{k_{1}}^{2} \xi_{k_{2}}^{2} \longrightarrow 1 \quad \text { при } n \rightarrow \infty .
$$

Из определения $\xi_{i}$ следует, что

$$
\xi_{k_{1}}^{2} \xi_{k_{2}}^{2}=B_{k_{1} k_{2}}^{(1)}+B_{k_{1} k_{2}}^{(2)}+B_{k_{1} k_{2}}^{(3)}+B_{k_{1} k_{2}}^{(4)}
$$


где

$$
\begin{aligned}
B_{k_{1} k_{2}}^{(1)} & =\sigma_{2}\left(k_{1}\right) \sigma_{2}\left(k_{2}\right), & B_{k_{1} k_{2}}^{(2)} & =\sigma_{2}\left(k_{1}\right) \sigma_{1}\left(k_{2}\right), \\
B_{k_{1} k_{2}}^{(3)} & =\sigma_{1}\left(k_{1}\right) \sigma_{2}\left(k_{2}\right), & B_{k_{1} k_{2}}^{(4)} & =\sigma_{1}\left(k_{1}\right) \sigma_{1}\left(k_{2}\right), \\
\sigma_{1}(k) & =\sum_{1 \leqslant i \neq j \leqslant k-1} \eta_{i k} \eta_{j k}, & \sigma_{2}(k) & =\sum_{i=1}^{k-1} \eta_{i k}^{2} .
\end{aligned}
$$

Следовательно,

$$
2 \sum_{1 \leqslant k_{1}<k_{2} \leqslant n} \mathbf{E} \xi_{k_{1}}^{2} \xi_{k_{2}}^{2}=\sum_{i=1}^{4} A_{n}^{(i)}
$$

где

$$
A_{n}^{(i)}=2 \sum_{1 \leqslant k_{1}<k_{2} \leqslant n} \mathbf{E} B_{k_{1} k_{2}}^{(i)}, \quad i=1,2,3,4 .
$$

Рассмотрим $A_{n}^{(3)}$. Используя определение $\eta_{i j}$, легко показать, что $\mathbf{E} B_{k_{1} k_{2}}^{(3)}=0$ и, следовательно,

$$
A_{n}^{(3)}=0
$$

Оценим $A_{n}^{(2)}$. Имеем

$$
\begin{aligned}
\left|\mathbf{E} B_{k_{1} k_{2}}^{(2)}\right|= & \frac{16}{n^{4} b_{n}^{4} \sigma_{n}^{4}}\left|\sum_{i=1}^{k_{1}-1} \mathbf{E} \varepsilon_{i}^{3} \mathbf{E} \varepsilon_{k_{1}}^{3} \mathbf{E} \varepsilon_{k_{2}}^{2} Q_{i k_{1}}^{2} Q_{i k_{2}} Q_{k_{1} k_{2}}\right| \\
= & \frac{16}{\left(n^{4} b_{n} \sigma_{n}\right)^{4}} \mid \sum_{i=1}^{k_{1}-1}\left(1-p_{i}\right) p_{i}\left(1-2 p_{i}\right)\left(1-p_{k_{1}}\right) p_{k_{1}}\left(1-2 p_{k_{1}}\right)\left(1-p_{k_{2}}\right) \\
& \quad \times p_{k_{2}}\left(1-2 p_{k_{2}}\right) Q_{i k_{1}}^{2} Q_{i k_{2}} Q_{k_{1} k_{2}} \mid \\
\leqslant & \frac{c_{4}\left(k_{1}-1\right)}{\left(n \sigma_{n}\right)^{4}} .
\end{aligned}
$$

Поскольку $\sum_{1 \leqslant k_{1}<k_{2} \leqslant n}\left(k_{1}-1\right)=O\left(n^{3}\right)$ и $b_{n}^{-1} \sigma_{n}^{2} \rightarrow \sigma^{2}(p)>0$, то отсюда получим

$$
\left|A_{n}^{(2)}\right| \leqslant \frac{c_{5} n^{3}}{n^{4} \sigma_{n}^{4}}=c_{5} \frac{1}{n b_{n}^{2}\left(b_{n}^{-1} \sigma_{n}^{2}\right)^{2}}=O\left(\frac{1}{n b_{n}^{2}}\right) .
$$

Теперь установим, что $A_{n}^{(1)} \rightarrow 1$ при $n \rightarrow \infty$. Очевидно, что

$$
A_{n}^{(1)}=2 \sum_{1 \leqslant k_{1}<k_{2} \leqslant n} \mathbf{E} B_{k_{1} k_{2}}^{(1)}=D_{n}^{(1)}+D_{n}^{(2)},
$$

где

$$
\begin{aligned}
& D_{n}^{(1)}=2 \sum_{1 \leqslant k_{1}<k_{2} \leqslant n}\left(\sum_{i=1}^{k_{1}-1} \mathbf{E} \eta_{i k_{1}}^{2}\right)\left(\sum_{j=1}^{k_{2}-1} \mathbf{E} \eta_{j k_{2}}^{2}\right) \\
& D_{n}^{(2)}=2\left(\sum_{k_{1}<k_{2}} \mathbf{E} B_{k_{1} k_{2}}^{(1)}-\sum_{k_{1}<k_{2}}\left(\sum_{i=1}^{k_{1}-1} \mathbf{E} \eta_{i k_{1}}^{2}\right)\left(\sum_{j=1}^{k_{2}-1} \mathbf{E} \eta_{j k_{2}}^{2}\right)\right)
\end{aligned}
$$


Из определения $\sigma_{n}^{2}$ следует, что

$$
D_{n}^{(1)}=1-\sum_{k=2}^{n}\left(\sum_{i=1}^{k-1} \mathbf{E} \eta_{i k}^{2}\right)^{2}
$$

Ho

$$
\sum_{k=2}^{n}\left(\sum_{i=1}^{k-1} \mathbf{E} \eta_{i k}^{2}\right)^{2} \leqslant c_{6} \frac{b_{n}^{4} n^{3}}{\left(n b_{n}\right)^{4} \sigma_{n}^{4}}=O\left(\frac{1}{n b_{n}^{2}}\right)
$$

Таким образом,

$$
D_{n}^{(1)} \rightarrow 1 \quad \text { при } n \rightarrow \infty \text {. }
$$

Далее, покажем, что $D_{n}^{(2)} \rightarrow 0$. Легко видеть, что

$$
D_{n}^{(2)}=2 \sum_{k_{1}<k_{2}}\left[\sum_{i=1}^{k_{1}-1} \operatorname{cov}\left(\eta_{i k_{1}}^{2}, \eta_{i k_{2}}^{2}\right)+\sum_{i=1}^{k_{1}-1} \operatorname{cov}\left(\eta_{i k_{1}}^{2}, \eta_{k_{1} k_{2}}^{2}\right)\right] .
$$

Ho

$$
\mathbf{E} \eta_{i k_{1}}^{2} \eta_{i k_{2}}^{2} \leqslant c_{7} \frac{Q_{i k_{1}}^{2} Q_{i k_{2}}^{2}}{\left(n b_{n}\right)^{4} \sigma_{n}^{4}} \leqslant c_{8} \frac{1}{n^{4} \sigma_{n}^{4}} .
$$

Точно так же

$$
\mathbf{E} \eta_{i j}^{2}=O\left(\frac{1}{n^{2} \sigma_{n}^{2}}\right)
$$

Значит,

$$
\operatorname{cov}\left(\eta_{i k_{1}}^{2}, \eta_{i k_{2}}^{2}\right)=O\left(\frac{1}{n^{4} b_{n}^{4}}\right) .
$$

Далее, поскольку $\sum_{1 \leqslant k_{1}<k_{2} \leqslant n}\left(k_{1}-1\right)=O\left(n^{3}\right)$, из (15) следует

$$
D_{n}^{(2)}=O\left(\frac{1}{n \sigma_{n}^{4}}\right)=O\left(\frac{1}{n b_{n}^{2}}\right) .
$$

Итак, согласно (14) и (16),

$$
A_{n}^{(1)}=1+O\left(\frac{1}{n b_{n}^{2}}\right) .
$$

Наконец, докажем, что $A_{n}^{(4)} \rightarrow 0$ при $n \rightarrow \infty$. Пользуясь определением $\eta_{i j}$ и соотношениями $Q_{i j} \geqslant 0$ и $\mathbf{E}\left(Y_{i}-p\left(x_{i}\right)\right)^{2}=p\left(x_{i}\right)\left(1-p\left(x_{i}\right)\right) \leqslant 1 / 4$, получаем

$$
\begin{aligned}
\left|\mathbf{E} B_{k_{1}, k_{2}}^{(4)}\right| & =4\left|\sum_{1 \leqslant t<s \leqslant k_{1}-1} \mathbf{E} \eta_{s k_{1}} \eta_{t k_{1}} \eta_{s k_{2}} \eta_{t k_{2}}\right| \\
& =4 \frac{1}{n^{4} b_{n}^{4} \sigma_{n}^{4}}\left|\sum_{1 \leqslant t<s \leqslant k_{1}-1} \mathbf{E} \varepsilon_{s}^{2} \varepsilon_{t}^{2} \varepsilon_{k_{1}}^{2} \varepsilon_{k_{2}}^{2} Q_{s k_{1}} Q_{t k_{1}} Q_{s k_{2}} Q_{t k_{2}}\right| \\
& \leqslant \frac{c_{8}}{n^{4} b_{n}^{4} \sigma_{n}^{4}} \sum_{1 \leqslant t<s \leqslant k_{1}-1} Q_{s k_{1}} Q_{t k_{1}} Q_{s k_{2}} Q_{t k_{2}} .
\end{aligned}
$$


Следовательно,

$$
\left|A_{n}^{(4)}\right| \leqslant \frac{c_{9}}{n^{2} b_{n}^{4} \sigma_{n}^{4}} \sum_{k_{1}<k_{2}} A_{k_{1} k_{2}}
$$

где

$$
A_{k_{1} k_{2}}=\frac{1}{n^{2}} \sum_{1 \leqslant t<s \leqslant k_{1}-1} Q_{s k_{1}} Q_{t k_{1}} Q_{s k_{2}} Q_{t k_{2}}
$$

Ho

$$
\begin{aligned}
\sum_{k_{1}<k_{2}} A_{k_{1} k_{2}} & \leqslant \frac{1}{n^{2}} \sum_{k_{1}, k_{2}=1}^{n} \sum_{t=1}^{n} \sum_{s=1}^{n} Q_{s k_{1}} Q_{t k_{1}} Q_{t k_{2}} Q_{s k_{2}} \\
& =\sum_{k_{1}, k_{2}=1}^{n}\left(\frac{1}{n} \sum_{t=1}^{n} Q_{t k_{1}} Q_{t k_{2}}\right)^{2} .
\end{aligned}
$$

Таким образом,

$$
\begin{aligned}
\left|A_{n}^{(4)}\right| \leqslant c_{9} \frac{1}{n^{2} b_{n}^{4} \sigma_{n}^{4}} \sum_{k_{1}, k_{2}=1}^{n}( & \left.\frac{1}{n} \sum_{t=1}^{n} Q_{t k_{1}} Q_{t k_{2}}\right)^{2} \\
=c_{9} \frac{1}{n^{2} b_{n}^{4} \sigma_{n}^{4}} \sum_{k_{1}, k_{2}=1}^{n}( & \sum_{i=1}^{n} \int_{\Omega_{n}(\tau)} K\left(\frac{x-x_{i}}{b_{n}}\right) K\left(\frac{x-x_{k_{1}}}{b_{n}}\right) d x \\
& \left.\times \int_{\Omega_{n}(\tau)} K\left(\frac{y-x_{i}}{b_{n}}\right) K\left(\frac{y-x_{k_{2}}}{b_{n}}\right) d y\right)^{2} \\
=c_{9} \frac{1}{n^{2} b_{n}^{4} \sigma_{n}^{4}} \sum_{k_{1}, k_{2}=1}^{n}[ & \int_{\Omega_{n}(\tau)} \int_{\Omega_{n}(\tau)} K\left(\frac{x-x_{k_{1}}}{b_{n}}\right) K\left(\frac{y-x_{k_{2}}}{b_{n}}\right)^{n} \\
& \left.\times \frac{1}{n} \sum_{i=1}^{n} K\left(\frac{x-x_{i}}{b_{n}}\right) K\left(\frac{y-x_{i}}{b_{n}}\right) d x d y\right]^{2} .
\end{aligned}
$$

Далее, воспользовавшись леммой 2 , из (18) можно заключить, что

$$
\begin{aligned}
\left|A_{n}^{(4)}\right| \leqslant & c_{9} \frac{1}{n^{2} b_{n}^{4} \sigma_{n}^{4}} \sum_{k_{1}, k_{2}=1}^{n}\{ \\
\times & \int_{\Omega_{n}(\tau)} \int_{\Omega_{n}(\tau)} K\left(\frac{x-x_{k_{1}}}{b_{n}}\right) K\left(\frac{y-x_{k_{2}}}{b_{n}}\right) d x d y \\
= & \left.c_{10} \frac{1}{b_{n}^{4} \sigma_{n}^{4}} \sum_{k_{1}, k_{2}=1}^{n}\left\{\frac{1}{n} \int_{0}^{1} \int_{\Omega_{n}(\tau)} \int_{\Omega_{n}(\tau)} K\left(\frac{x-u}{b_{n}}\right) K\left(\frac{y-u}{b_{n}}\right) d u+O\left(\frac{1}{n}\right)\right]\right\}^{2} \\
& \left.\times K\left(\frac{x-u}{b_{n}}\right) K\left(\frac{y-u}{b_{n}}\right) d u d x d y\right\}^{2} \\
+ & O\left(\frac{1}{n b_{n}^{2}}\right) .
\end{aligned}
$$


Аналогично, если в (19) снова применить лемму 2, то можно показать, что

$$
\begin{aligned}
\left|A_{n}^{(4)}\right| \leqslant & \frac{c_{10}}{b_{n}^{4} \sigma_{n}^{4}} \int_{0}^{1} \int_{0}^{1} \int_{0}^{1} \int_{0}^{1} \psi_{n}\left(u_{1}, v_{2}\right) \psi_{n}\left(u_{1}, v_{1}\right) \\
& \times \psi_{n}\left(u_{2}, v_{1}\right) \psi_{n}\left(u_{2}, v_{2}\right) d u_{1} d u_{2} d v_{1} d v_{2} \\
& +O\left(\frac{1}{n b_{n}^{2}}\right)
\end{aligned}
$$

где

$$
\psi_{n}(x, y)=\int_{\Omega_{n}(\tau)} K\left(\frac{u-x}{b_{n}}\right) K\left(\frac{u-y}{b_{n}}\right) d u .
$$

Теперь оценим интеграл $I_{n}$, входящий в (20). Имеем

$$
I_{n}=\int_{0}^{1} \int_{0}^{1} \int_{0}^{1} \psi_{n}\left(u_{2}, v_{1}\right) \psi_{n}\left(u_{2}, v_{2}\right) d v_{1} d v_{2} d u_{2} \int_{0}^{1} \psi_{n}\left(u_{1}, v_{2}\right) \psi_{n}\left(u_{1}, v_{1}\right) d u_{1}
$$

Но поскольку $\left[(x-1) / b_{n}, x / b_{n}\right] \supseteq[-\tau, \tau]$ для всех $x \in \Omega_{n}(\tau)$, то

$$
\begin{aligned}
& \int_{0}^{1} \psi_{n}\left(u_{1}, v_{2}\right) \psi_{n}\left(u_{1}, v_{1}\right) d u_{1} \\
& =b_{n} \int_{\Omega_{n}(\tau)} \int_{\Omega_{n}(\tau)} K\left(\frac{t-v_{2}}{b_{n}}\right) K\left(\frac{z-v_{1}}{b_{n}}\right) d t d z \int_{(t-1) / b_{n}}^{t / b_{n}} K(\xi) K\left(\frac{t-z}{b_{n}}-\xi\right) d \xi \\
& =b_{n} \int_{\Omega_{n}(\tau)} \int_{\Omega_{n}(\tau)} K\left(\frac{t-v_{2}}{b_{n}}\right) K\left(\frac{z-v_{1}}{b_{n}}\right) K_{0}\left(\frac{z-t}{b_{n}}\right) d t d z \\
& \leqslant c_{11} b_{n}^{3}, \quad K_{0}=K * K
\end{aligned}
$$

Следовательно,

$$
\left|A_{n}^{(4)}\right| \leqslant c_{12} \frac{1}{b_{n} \sigma_{n}^{4}} \int_{0}^{1} \int_{0}^{1} \int_{0}^{1} \psi_{n}\left(u_{2}, v_{1}\right) \psi_{n}\left(u_{2}, v_{2}\right) d u_{2} d v_{1} d v_{2}+O\left(\frac{1}{n b_{n}^{2}}\right) .
$$

Повторяя те же рассуждения для интеграла, входящего в (21), окончательно получим

$$
\begin{aligned}
\left|A_{n}^{(4)}\right| & \leqslant c_{13} \frac{b_{n}^{4}}{b_{n} \sigma_{n}^{4}}+O\left(\frac{1}{n b_{n}^{2}}\right)=O\left(\frac{b_{n}^{4}}{b_{n}^{3}\left(b_{n}^{-1} \sigma_{n}^{2}\right)^{2}}\right)+O\left(\frac{1}{n b_{n}^{2}}\right) \\
& =O\left(b_{n}\right)+O\left(\frac{1}{n b_{n}^{2}}\right) .
\end{aligned}
$$

Собрав вместе соотношения (12), (13), (17) и (22), заключаем, что

$$
2 \sum_{1 \leqslant k_{1}<k_{2} \leqslant n} \mathbf{E} \xi_{k_{1}}^{2} \xi_{k_{2}}^{2} \longrightarrow 1 \text {. }
$$


Отсюда и из (11) вытекает, что

$$
\mathbf{E}\left(\sum_{k=1}^{n} \xi_{k}^{2}-1\right)^{2} \longrightarrow 0 \quad \text { при } n \rightarrow \infty .
$$

Стало быть,

$$
\frac{U_{n}-\Delta_{n}}{\sigma_{n}} \stackrel{d}{\longrightarrow} N(0,1)
$$

Далее, используя лемму 3 , из (23) находим

$$
b_{n}^{-1 / 2} \frac{U_{n}-\Delta(p)}{\sigma(p)} \stackrel{d}{\longrightarrow} N(0,1) .
$$

Теорема 1 доказана.

Теорема 2. Пусть $K(x) \in H(\tau), p(x) \in C^{(2)}$. Тогда, если $n b_{n}^{2} \rightarrow \infty$ $u n b_{n}^{4} \rightarrow 0, m o$

$$
b_{n}^{-1 / 2} \frac{T_{n}-\Delta(\rho)}{\sigma(p)} \stackrel{d}{\longrightarrow} N(0,1) .
$$

Д ок аз а т е л ь с т в о. Имеем $T_{n}=U_{n}+R_{n}^{(1)}+R_{n}^{(2)}$, где

$$
\begin{aligned}
& R_{n}^{(1)}=2 n b_{n} \int_{\Omega_{n}(\tau)}\left[p_{1 n}(x)-\mathbf{E} p_{1 n}(x)\right]\left[\mathbf{E} p_{1 n}(x)-p_{2 n}(x) p(x)\right] d x, \\
& R_{n}^{(2)}=n b_{n} \int_{\Omega_{n}(\tau)}\left[\mathbf{E} p_{1 n}(x)-p_{2 n}(x) p(x)\right]^{2} d x .
\end{aligned}
$$

Легко видеть, что

$$
\mathbf{E} p_{1 n}(x)=\frac{1}{n b_{n}} \sum_{i=1}^{n} K\left(\frac{x-x_{i}}{b_{n}}\right) p\left(x_{i}\right) .
$$

Отсюда в силу леммы 2 следует, что

$$
\begin{aligned}
\mathbf{E} p_{1 n}(x) & =\frac{1}{b_{n}} \int_{0}^{1} K\left(\frac{x-u}{b_{n}}\right) p(u) d u+O\left(\frac{1}{n b_{n}}\right) \\
& =\int_{(x-1) / b_{n}}^{x / b_{n}} K(t) p\left(x-b_{n} t\right) d t+O\left(\frac{1}{n b_{n}}\right) .
\end{aligned}
$$

Поскольку $p(x) \in C^{(2)}$ и $\left[(x-1) / b_{n}, x / b_{n}\right] \supset[-\tau, \tau]$ для всех $x \in \Omega_{n}(\tau)$, то из $(24)$ находим

$$
\mathbf{E} p_{1 n}(x)=p(x)+O\left(b_{n}^{2}\right)+O\left(\frac{1}{n b_{n}}\right) .
$$

Далее, согласно лемме 2,

$$
\begin{aligned}
p_{2 n}(x) & =\frac{1}{b_{n}} \int_{0}^{1} K\left(\frac{x-u}{b_{n}}\right) d u+O\left(\frac{1}{n b_{n}}\right) \\
& =\int_{-\tau}^{\tau} K(u) d u+O\left(\frac{1}{n b_{n}}\right)=1+O\left(\frac{1}{n b_{n}}\right) .
\end{aligned}
$$


Из (24), (25) и (26) вытекает, что

$$
b_{n}^{-1 / 2} R_{n}^{(2)} \leqslant c_{14}\left(n b_{n}^{9 / 2}+b_{n}^{3 / 2}+\frac{1}{n b_{n}^{3 / 2}}\right) \longrightarrow 0 .
$$

Оценим теперь $b_{n}^{-1 / 2} \mathbf{E}\left|R_{n}^{(1)}\right|$. Из (25) и (26) следует неравенство

$$
b_{n}^{-1 / 2} \mathbf{E}\left|R_{n}^{(1)}\right| \leqslant c_{15} n b_{n}^{1 / 2}\left[b_{n}^{2}+\frac{1}{n b_{n}}\right] \int_{\Omega_{n}(\tau)}\left(\mathbf{E}\left[p_{1 n}(x)-\mathbf{E} p_{1 n}(x)\right]^{2}\right)^{1 / 2} d x .
$$

Но, согласно лемме 2, имеем

$$
\begin{aligned}
\mathbf{E}\left[p_{1 n}(x)-\mathbf{E} p_{1 n}(x)\right]^{2}= & \frac{1}{n b_{n}} p(x)(1-p(x)) \int_{|u| \leqslant \tau} K^{2}(u) d u \\
& +O\left(\frac{1}{\left(n b_{n}\right)^{2}}\right)+O\left(\frac{1}{n}\right) .
\end{aligned}
$$

Отсюда и из (28) находим, что

$$
b_{n}^{-1 / 2} \mathbf{E}\left|R_{n}^{(1)}\right| \leqslant c_{16}\left(\sqrt{n} b_{n}^{2}+\frac{1}{\sqrt{n} b_{n}}\right) \longrightarrow 0 .
$$

Утверждение теоремы 2 непосредственно вытекает из теоремы 1 и соотношений (27) и (29).

4. Применение статистики $T_{n}$ для проверки гипотезы. Утверждение теоремы 2 позволяет конструировать критерий асимптотического уровня $\alpha, 0<\alpha<1$, для проверки гипотезы $H_{0}$, согласно которой $p(x)=p_{0}(x), x \in \Omega_{n}(\tau)$. Критическая область устанавливается неравенством

$$
T_{n} \geqslant q_{n}(\alpha)
$$

где

$$
\begin{aligned}
q_{n}(\alpha) & =\Delta\left(p_{0}\right)+\lambda_{\alpha} \sqrt{b_{n}} \sigma\left(p_{0}\right), \\
\Delta\left(p_{0}\right) & =\int_{0}^{1} p_{0}(x)\left(1-p_{0}(x)\right) d x \int_{|u| \leqslant \tau} K^{2}(u) d u, \\
\sigma^{2}\left(p_{0}\right) & =2 \int_{0}^{1} p_{0}^{2}(x)\left(1-p_{0}^{2}(x)\right) d x \int_{|u| \leqslant 2 \tau} K_{0}^{2}(u) d u,
\end{aligned}
$$

а $\lambda_{\alpha}$ определяется равенством $\Phi\left(\lambda_{\alpha}\right)=1-\alpha$.

Теперь исследуем асимптотические свойства критерия (30) (т.е. поведение функции мощности при $n \rightarrow \infty)$. Прежде всего рассмотрим вопрос, является ли критерий состоятельным. Справедливо следующее утверждение. 
Теорема 3. Пусть выполняются все условия теоремь 2. Тогда

$$
\Pi_{n}(p)=\mathbf{P}_{H_{1}}\left\{T_{n} \geqslant q_{n}(\alpha)\right\} \longrightarrow 1 \text { npu } n \rightarrow \infty,
$$

т.е. критерий, определенный в (30), состоятелен против любой альтернативь $H_{1}: p(x) \neq p_{0}(x), 0 \leqslant x \leqslant 1$.

Д о к а з а т ел ь с т в о. Обозначим

$$
z_{n}(p)=b_{n}^{-1 / 2}\left(n b_{n} \int_{\Omega_{n}(\tau)}\left[\widehat{p}_{n}(x)-p(x)\right]^{2} p_{2 n}^{2}(x) d x-\Delta(p)\right) \sigma^{-1}(p) .
$$

Нетрудно показать, что

$$
\Pi_{n}(p)=\mathbf{P}_{H_{1}}\left\{z_{n}(p) \geqslant-n b_{n}^{1 / 2}\left(\int_{0}^{1}\left(p(x)-p_{0}(x)\right)^{2} d x+o_{\mathbf{P}}(1)\right)\right\} .
$$

Поскольку $z_{n}(p)$ распределена асимптотически нормально с параметрами $(0,1)$ при гипотезе $H_{1}$ и $n b_{n}^{1 / 2} \rightarrow \infty$, то $\Pi_{n}(p) \rightarrow 1$ при $n \rightarrow \infty$. Теорема 3 доказана.

Таким образом, мощность критерия, основанного на $T_{n}$, при любой фиксированной альтернативе стремится к 1. Однако, если при изменении $n$ альтернатива изменяется, приближаясь к основной гипотезе $H_{0}$, то мощность критерия уже не обязательно будет сходиться к 1. Так, например, рассмотрим последовательность близких к гипотезе $H_{0}$ альтернатив типа Питмена:

$$
H_{1}: \quad p_{1}^{(n)}(x)=p_{0}(x)+\gamma_{n} \varphi(x)+o\left(\gamma_{n}\right), \quad \gamma_{n} \rightarrow 0 .
$$

Теорема 4. Пусть $p_{0}(x), \varphi(x) \in C^{(2)}, a K(x) \in H(\tau) . \quad$ Если $b_{n}=n^{-\delta}, \gamma_{n}=n^{-1 / 2+\delta / 4}, 1 / 4<\delta<1 / 2$, то статистика $b_{n}^{-1 / 2}\left(T_{n}-\right.$ $\left.\Delta\left(p_{0}\right)\right) \sigma^{-1}\left(p_{0}\right)$ при альтернативе $H_{1}$ распределена в пределе нормально с параметрами

$$
\left(\frac{1}{\sigma\left(p_{0}\right)} \int_{0}^{1} \varphi^{2}(x) d x, 1\right)
$$

т.е. предельная мощность критерия равна

$$
1-\Phi\left(\lambda_{\alpha}-\frac{1}{\sigma\left(p_{0}\right)} \int_{0}^{1} \varphi^{2}(u) d u\right) .
$$

Д о к а з а т е л ь с т в о. Представим $T_{n}$ в виде

$$
\begin{aligned}
T_{n}= & n b_{n} \int_{\Omega_{n}(\tau)}\left(\widehat{p}(x)-p_{1}^{(n)}(x)\right)^{2} p_{2 n}^{2}(x) d x \\
& +n b_{n} \int_{\Omega_{n}(\tau)}\left(p_{1}^{(n)}(x)-p_{0}(x)\right)^{2} p_{2 n}^{2}(x) d x \\
& +2 n b_{n} \int_{\Omega_{n}(\tau)}\left(\widehat{p}_{n}(x)-p_{1}^{(n)}(x)\right)\left(p_{1}^{(n)}(x)-p_{0}(x)\right)^{2} p_{2 n}^{2}(x) d x \\
= & T_{n}^{*}+A_{1}(n)+A_{2}(n) .
\end{aligned}
$$


Поскольку $p_{2 n}(x)=1+O\left(1 /\left(n b_{n}\right)\right)$ равномерно по $x \in \Omega_{n}(\tau)$, то

$$
b_{n}^{-1 / 2} A_{1}(n)=\int_{0}^{1} \varphi^{2}(u) d u+o(1)
$$

Обозначим

$$
D_{n}=\int_{\Omega_{n}(\tau)}\left(p_{1 n}(x)-\mathbf{E}_{1} p_{1 n}(x)\right) \varphi(x) p_{2 n}(x) d x,
$$

где $\mathbf{E}_{1}$ - математическое ожидание при гипотезе $H_{1}$. Тогда

$$
\begin{aligned}
b_{n}^{-1 / 2} A_{2}(n) & =n b_{n}^{1 / 2} \gamma_{n} D_{n}+n b_{n}^{1 / 2} \gamma_{n}\left(b_{n}^{2}+O\left(\frac{1}{n b_{n}}\right)\right) \\
& =n b_{n}^{1 / 2} \gamma_{n} D_{n}+O\left(n b_{n}^{5 / 2} \gamma_{n}\right)+O\left(\frac{\gamma_{n}}{\sqrt{b_{n}}}\right) .
\end{aligned}
$$

Теперь покажем, что $n b_{n}^{1 / 2} \gamma_{n} D_{n} \stackrel{\mathbf{P}}{\longrightarrow} 0$. В самом деле,

$$
\begin{aligned}
\mathbf{E}\left|D_{n}\right| & \leqslant\left(\mathbf{E} D_{n}^{2}\right)^{1 / 2}=\left\{\frac{1}{n b_{n}} \int_{\Omega_{n}(\tau)} \int_{\Omega_{n}(\tau)} p_{2 n}(x) p_{2 n}(y) d x d y\right. \\
\times & \left.\frac{1}{n b_{n}} \sum_{i=1}^{n} K\left(\frac{x-x_{i}}{b_{n}}\right) K\left(\frac{y-x_{i}}{b_{n}}\right) p_{1}^{(n)}\left(x_{i}\right)\left(1-p_{1}^{(n)}\left(x_{i}\right)\right)\right\}^{1 / 2} .
\end{aligned}
$$

Далее, используя лемму 2 , нетрудно убедиться, что

$$
\begin{aligned}
\mathbf{E}\left|D_{n}\right| \leqslant\{ & \frac{1}{n b_{n}} \int_{\Omega_{n}(\tau)} \int_{\Omega_{n}(\tau)} p_{2 n}(x) p_{2 n}(y) d x d y \\
& \times\left[\frac{1}{b_{n}} \int_{0}^{1} K\left(\frac{u}{b_{n}}\right) K\left(\frac{y-u}{b_{n}}\right) p_{1}^{(n)}(u)\left(1-p_{1}^{(n)}(u)\right) d u\right. \\
& \left.\left.+O\left(\frac{1}{n b_{n}}\right)\right]\right\}^{1 / 2} \leqslant c_{17} \frac{1}{\sqrt{n}}\left(1+\frac{1}{n b_{n}^{2}}\right) .
\end{aligned}
$$

Стало быть, $n b_{n}^{1 / 2} \gamma_{n} \mathbf{E}\left|D_{n}\right| \leqslant c_{18} n^{-\delta / 4}$. Значит,

$$
n b_{n}^{1 / 2} \gamma_{n} D_{n} \stackrel{\mathbf{P}}{\longrightarrow} 0 .
$$

Далее, случайная величина $b_{n}^{-1 / 2}\left(T_{n}^{*}-\Delta\left(p_{1}^{(n)}\right)\right) \sigma^{-1}\left(p_{1}^{(n)}\right)$ асимптотически нормальна со средним 0 и дисперсией 1 . Отсюда и из $(32),(33)$ и $(34)$ следует доказательство теоремы 4.

3 а м е ч а н и е 1 . Стоит подчеркнуть, что оценка $\widehat{p}_{n}(x)$ около границы интервала $[0,1]$ ведет себя хуже, чем во внутренном интервале $\left[\tau b_{n}, 1-\tau b_{n}\right]$ (см. [9]). Поэтому мы рассматриваем интегральное квадратическое уклонение на $\Omega_{n}(\tau)$ - для того чтобы избежать трудностей, связанных с указанным граничным эффектом; однако можно показать, 
что при условиях теоремы 1 и 2 полученные выше результаты справедливы и для модифицированной оценки (см. [9], [10]) функции $p(x)$.

3 а м е ч а н и е 2. Результаты, близкие к теоремам 1 и 2, приведены в работах [11] и [12]. К сожалению, в доказательстве основной теоремы 1 из [11] имеются некоторые неточности [11, с. 92]. Кроме того, в ней не установлена в явном виде асимптотика нормирующих величин, что затрудняет применение этой теоремы для задач статистики.

3 а м е ч а н и е 3 . Пусть $x_{i}$ - точки деления интервала $[0,1]$, выбранные так, что $H\left(x_{j}\right)=(2 j-1) /(2 n), j=1, \ldots, n$, где $H(x)=$ $\int_{0}^{x} h(u) d u, h(u)$ - какая-нибудь известная непрерывная плотность распределения на $[0,1]$. В этом случае рассуждениями, аналогичными приводимым выше, можно получить обобщение результатов данной работы.

\section{СПИСОК ЛИТЕРАТУРЫ}

1. Efromovich S. Nonparametric Curve Estimation. Methods, Theory, and Applications. New York: Springer-Verlag, 1999, $411 \mathrm{p}$.

2. Copas J. B. Plotting $p$ against $x$. - Appl. Statist., 1983, v. 32, № 1, p. 25-31.

3. Okumura H., Naito K. Weighted kernel estimators in nonparametric binomial regression. - J. Nonparametr. Statist., 2004, v. 16, № 1-2, p. 39-62.

4. Манджаладзе К. В. Об одной оценке функции распределения и ее моментов. Сообщ. АН ГрССР, 1986, т. 124, № 2, с. 261-263.

5. Надарая Э. А. Об оценке регрессии. - Теория вероятн. и ее примен., 1964, т. 9, B. 1, c. $157-159$.

6. Watson G. S. Smooth regression analysis. - Sankhyā Ser. A, 1964, v. 26, p. 359-372.

7. Nadaraya E., Babilua P., Sokhadze G. Estimation of a distribution function by an indirect sample. - Укр. матем. журн., 2010, т. 62, № 12, с. 1642-1658.

8. Липцер P. Ш., Ширяев A.Н. Функциональная центральная предельная теорема для семимартингалов. - Теория вероятн. и ее примен., 1980, т. 25 , в. 4 , с. $683-$ 703.

9. Hart J. D., Wehrly Th. E. Kernel regression when the boundary region is large, with an application to testing the adequacy of polynomial models. - J. Amer. Statist. Assoc., 1992, v. 87, № 420, p. 1018-1024.

10. Абсава Р.М., Надарая Э.А. Некоторые задачи теории непараметрического оценивания функциональных характеристик закона распределения наблюдений. Тбилиси: Изд-во Тбил. ун-та, 2005, 247 с.

11. Ioannides D.A. Integrated square error of nonparametric estimators of regression function: the fixed design case. - Statist. Probab. Lett., 1992, v. 15, № 2, p. 85-94.

12. Absava R., Nadaraya E. On quadratic measure of deviation of non-parametric estimation of the Gasser-Müller regression function. - Proc. A. Razmadze Math. Inst., 2000, v. 122, p. 1-14. 